\title{
A New Direct Power Control Strategy for NPC Three-Level Voltage Source Rectifiers Using a Novel Vector Influence Table Method
}

\author{
Chang-Liang $\mathrm{Xia}^{\dagger}$, Zhe $\mathrm{Xu}^{*}$, and Jia-Xin Zhao ${ }^{*}$ \\ ${ }^{\dagger *}$ School of Electrical Engineering and Automation, Tianjin University, Tianjin, China
}

\begin{abstract}
This paper proposes a novel direct power control (DPC) strategy for neutral-point-clamped (NPC) three-level rectifiers, to directly control the active power, the reactive power and the neutral point potential of the rectifiers by referring to three pre-calculated vector influence tables and minimizing an objective function. In the three vector influence tables, the influences of different voltage vectors on the active power, the reactive power and the neutral-point potential are shown explicitly. A conceptual description and control algorithm of the proposed controller are presented in this paper. Then, numerical simulations and experiments are carried out to validate the proposed method. Both the simulation and experimental results show that good performances during both the steady-state and transient operating conditions are achieved. As a result, the proposed strategy has been proven to be effective for NPC three-level rectifiers.
\end{abstract}

Key words: Active power, Direct power control (DPC), Neutral-point potential, NPC three-level rectifiers, Reactive power

\section{INTRODUCTION}

Neutral-point-clamped (NPC) three-level rectifiers have been widely used in medium power applications since Nabae et al. proposed the topology in 1981. This is due to the fact that it has a number of advantages such as low harmonic distortion of the input currents, low switching voltage stress and low $\mathrm{d} u / \mathrm{d} t[1]-[6]$.

Although the three-level NPC topology provides significant advantages over the conventional two-level topology in high-power applications, it still has some inherent problems which have limited its practical application such as the voltage drifts and voltage ripples of the neutral-point. Therefore, many hardware and software methods have been proposed for balancing the neutral-point potential.

The high-performance control strategies of three-level voltage source PWM rectifiers are mainly the voltage oriented control (VOC) [7] and direct power control (DPC) [8], which are similar to the vector control (VC) and direct

Manuscript received Jun. 3, 2014; accepted Aug. 26, 2014

Recommended for publication by Associate Editor Liqiang Yuan.

${ }^{\dagger}$ Corresponding Author: motor@ $@$ tju.edu.cn

Tel: +86-22-27402325, Fax: ++86-22-27402325, Tianjin University

*School of Electrical Eng. and Automation, Tianjin University, China torque control (DTC) [9] for AC machines. The VOC guarantees high dynamic and steady-state performances via internal current control loops. This scheme decouples the converter currents into active and reactive power components The control of the active and reactive powers is then achieved by controlling the decoupled converter currents using current controllers. One main drawback of such a system is that the performance is highly dependent on the applied current control strategy and the connected AC network conditions [10]. The DPC strategy is based on the evaluation of active and reactive instantaneous power error values to choose appropriate voltage vectors to control the active and reactive power. When compared with the VOC strategy, DPC does not need internal current control loops or separate PWM modules. It has a simple control algorithm, fast dynamic response and high efficiency. However, since an optimal switching table is essential to the conventional DPC system, most of the switching tables of three-level rectifiers are established on the basis of qualitative analysis, without providing the quantitative influence of each space voltage vector on the active and reactive powers in each sector. Therefore, the performance of power control with the conventional switching table method is unsatisfactory.

There has been literature lot of research on how to 
overcome the shortcomings of the conventional DPC strategy. The three main methods are listed as follows:

1) Using multi-level hysteresis instead of two-level hysteresis. Four-level [11] and five-level [12] hysteresis DPC are proposed to increase the flexibility in terms of selecting an appropriate voltage vector, but they are still relatively rough and use qualitative control instead of quantitative control.

2) Using SVM instead of a switching table. DPC-SVM strategies usually calculate their output voltage references by means of closed-loop schemes with PI controllers [13]-[15], with errors of the active and reactive powers as the input of the controller. The power can be adjusted accurately and smoothly, and a constant switching frequency can be achieved. The main drawback of such a system is that the performance is highly dependent on the tuning of the PI controller.

3) Using predictive direct power control (P-DPC). Rodriguez et al. proposed a new strategy that eliminates the hysteresis controllers and switching table [16], [17]. In the proposed control strategy, the effects of each switching state on the input power are evaluated, and the switching state that minimizes a quality function is selected and applied during the next sampling period. However, since most P-DPC methods require a large amount of on-line computation in every control cycle, how to reduce the computational burden has become a major issue for P-DPC.

In this paper, a novel direct power control (DPC) strategy is proposed for NPC three-level rectifiers. In order to control the active and reactive power and the neutral point potential, three pre-calculated vector influence tables are established and an optimized switching state is selected by an objective function. This paper is organized as follows. Section II introduces the three-level PWM rectifier model and the neutral-point potential. The influences of different voltage vectors on the active and reactive power and the neutral-point potential are analyzed in Section III. The vector influence tables and the novel DPC algorithm are proposed in Section IV. Simulation and experimental studies are carried out in Section V and VI. Finally, some conclusions are summarized in Section VII.

\section{THREE-LEVEL VOLTAGE-SOURCE RECTIFIER}

\section{A. Model of a Three-Level PWM Rectifier}

A typical NPC three-level voltage-source rectifier is shown in Fig. 1. $e_{\mathrm{a}}, e_{\mathrm{b}}, e_{\mathrm{c}}, i_{\mathrm{a}}, i_{\mathrm{b}}$, and $i_{\mathrm{c}}$ are the three-phase grid voltages and currents, and $i_{0}$ is the neutral-point current. $L$ and $R$ represent the $\mathrm{AC}$-side line inductance and equivalent resistance, respectively. $C_{1}$ and $C_{2}$ are the capacitances of the DC-bus's upper and lower capacitors, whose voltages are $U_{\mathrm{dc} 1}$ and $U_{\mathrm{dc} 2}$.

The switching function $S_{j}\left(S_{j}=1,0\right.$ and $\left.-1, j=\mathrm{a}, \mathrm{b}, \mathrm{c}\right)$ is introduced to describe the states of the four switching devices

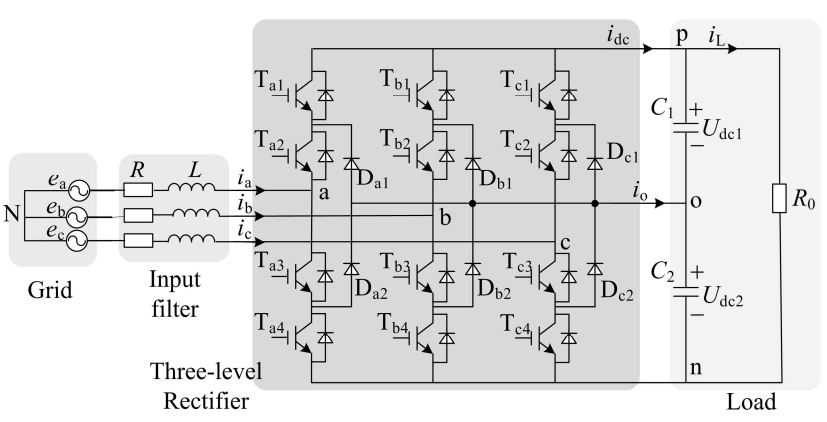

Fig. 1. Main circuit of a NPC three-level voltage-source rectifier.

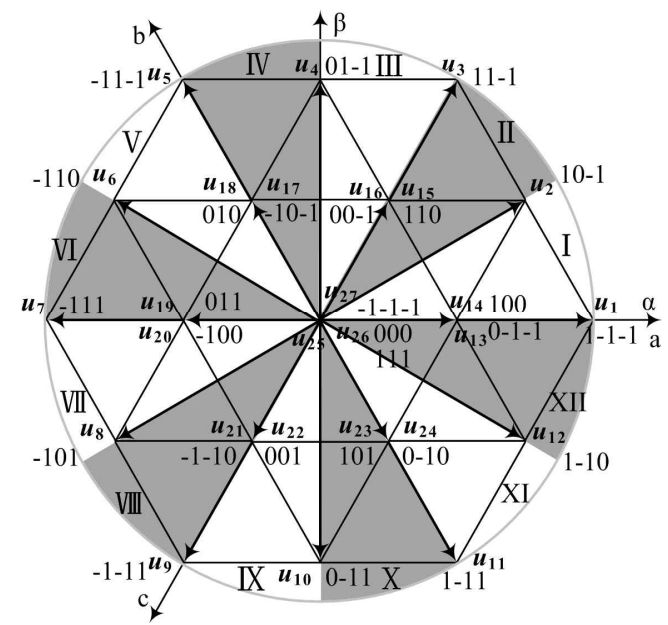

Fig. 2. Space voltage vectors of NPC three-level rectifiers.

TABLE I

Voltage Vectors of NPC THREe-LEVEl RECTIFIERS

\begin{tabular}{|c|c|c|}
\hline Type & Voltage vectors & Magnitude \\
\hline $\boldsymbol{u}_{\mathrm{L}}$ & $\boldsymbol{u}_{\mathbf{1}} \boldsymbol{u}_{\mathbf{3}} \boldsymbol{u}_{\mathbf{5}} \boldsymbol{u}_{\mathbf{7}} \boldsymbol{u}_{\mathbf{9}} \boldsymbol{u}_{\mathbf{1 1}}$ & $\sqrt{2 / 3} U_{\mathrm{dc}}$ \\
\hline $\boldsymbol{u}_{\mathbf{M}}$ & $\boldsymbol{u}_{\mathbf{2}} \boldsymbol{u}_{\mathbf{4}} \boldsymbol{u}_{\mathbf{6}} \boldsymbol{u}_{\mathbf{8}} \boldsymbol{u}_{\mathbf{1 0}} \boldsymbol{u}_{\mathbf{1 2}}$ & $\sqrt{1 / 2} U_{\mathrm{dc}}$ \\
\hline $\boldsymbol{u}_{\mathrm{S}}$ & $\begin{array}{c}\boldsymbol{u}_{13} \boldsymbol{u}_{15} \boldsymbol{u}_{\mathbf{1 7}} \boldsymbol{u}_{\mathbf{1 9}} \boldsymbol{u}_{\mathbf{2 1}} \boldsymbol{u}_{\mathbf{2 3}} \\
\boldsymbol{u}_{\mathbf{1 4}} \boldsymbol{u}_{\mathbf{1 6}} \boldsymbol{u}_{\mathbf{1 8}} \boldsymbol{u}_{\mathbf{2 0}} \boldsymbol{u}_{\mathbf{2 2}} \boldsymbol{u}_{\mathbf{2 4}}\end{array}$ & $\sqrt{1 / 6} U_{\mathrm{dc}}$ \\
\hline $\boldsymbol{u}_{\mathrm{Z}}$ & $\boldsymbol{u}_{\mathbf{2 5}} \boldsymbol{u}_{\mathbf{2 6}} \boldsymbol{u}_{\mathbf{2 7}}$ & 0 \\
\hline
\end{tabular}

of each phase. Taking phase a as an example, when $\mathrm{T}_{\mathrm{a} 1}$ and $\mathrm{T}_{\mathrm{a} 2}$ are on, $S_{\mathrm{a}}=1$, and the voltage between point a to point $\mathrm{o}$ is $U_{\mathrm{dc}} / 2$. When $\mathrm{T}_{\mathrm{a} 2}$ and $\mathrm{T}_{\mathrm{a} 3}$ are on, $S_{\mathrm{a}}=0$, and the voltage between point a to point $\mathrm{o}$ is 0 . When $\mathrm{T}_{\mathrm{a} 3}$ and $\mathrm{T}_{\mathrm{a} 4}$ are on, $S_{\mathrm{a}}=-1$, and the voltage between point a to point o is $-U_{\mathrm{dc}} / 2$. As a result, each phase voltage has three levels, $U_{\mathrm{dc}} / 2,0$ and $-U_{\mathrm{dc}} / 2$, leading to $3^{3}=27$ space voltage vectors for a three-phase three-level rectifier, as shown in Fig. 2. The space is divided into 12 zones according to the positions of the space voltage vectors. Based on their magnitudes, these vectors can be divided into four groups: zero vectors $\left(\boldsymbol{u}_{\mathrm{Z}}\right)$, small vectors $\left(\boldsymbol{u}_{\mathbf{S}}\right)$, medium vectors $\left(\boldsymbol{u}_{\mathbf{M}}\right)$, and large vectors $\left(\boldsymbol{u}_{\mathbf{L}}\right)$. The magnitudes of the different voltage vectors are listed in Table I.

According to Kirchhoff's voltage law, the mathematical model in the $d-q$ rotating reference frame can be expressed by: 


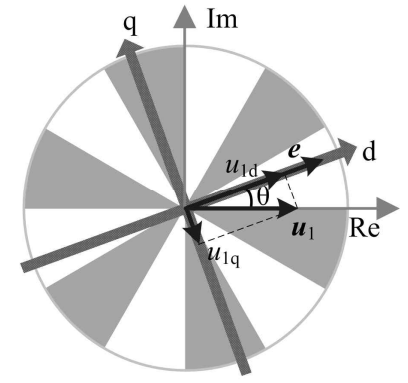

(a)

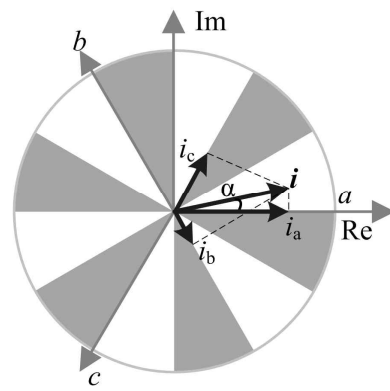

(b)
Fig. 3. (a) Space voltage vector projected on d-q frame. (b) AC current vector projected on abc frame.

$$
\left\{\begin{array}{l}
L \frac{\mathrm{d} i_{\mathrm{d}}}{\mathrm{d} t}=e_{\mathrm{d}}-u_{\mathrm{d}}-R i_{\mathrm{d}}+\omega L i_{\mathrm{q}} \\
L \frac{\mathrm{d} i_{\mathrm{q}}}{\mathrm{d} t}=e_{\mathrm{q}}-u_{\mathrm{q}}-R i_{\mathrm{q}}-\omega L i_{\mathrm{d}}
\end{array}\right.
$$

where $e_{\mathrm{d}}, e_{\mathrm{q}}, i_{\mathrm{d}}, i_{\mathrm{q}}, u_{\mathrm{d}}$ and $u_{\mathrm{q}}$ are the grid voltages, AC currents and rectifier input voltages of the PWM rectifier in the d-q coordinates, respectively. $\omega$ is the grid voltage angular velocity.

The expressions of the instantaneous active power $p$ and the reactive power $q$ can be calculated as follows:

$$
\left\{\begin{array}{l}
p=e_{\mathrm{d}} i_{\mathrm{d}}+e_{\mathrm{q}} i_{\mathrm{q}} \\
q=e_{\mathrm{q}} i_{\mathrm{d}}-e_{\mathrm{d}} i_{\mathrm{q}}
\end{array}\right.
$$

In the d-q rotating coordinates, the vector of three-phase grid voltages can be expressed as:

$$
\left\{\begin{array}{l}
e_{\mathrm{d}}=E_{1} \\
e_{\mathrm{q}}=0
\end{array}\right.
$$

where $E_{1}$ is the rms of the grid line voltage.

The expression of the instantaneous active power $p$ and the reactive power $q$ can be simplified as follows:

$$
\left\{\begin{array}{l}
p=E_{1} i_{\mathrm{d}} \\
q=-E_{1} i_{\mathrm{q}}
\end{array}\right.
$$

By multiplying both sides of equation (1) by $e_{\mathrm{d}}$, the following expressions can be obtained:

$$
\left\{\begin{array}{l}
L \frac{\mathrm{d} i_{\mathrm{d}}}{\mathrm{d} t} e_{\mathrm{d}}=e_{\mathrm{d}}^{2}-u_{\mathrm{d}} e_{\mathrm{d}}-R i_{\mathrm{d}} e_{\mathrm{d}}+\omega L i_{\mathrm{q}} e_{\mathrm{d}} \\
L \frac{\mathrm{d} i_{\mathrm{q}}}{\mathrm{d} t} e_{\mathrm{d}}=e_{\mathrm{q}} e_{\mathrm{d}}-u_{\mathrm{q}} e_{\mathrm{d}}-R i_{\mathrm{q}} e_{\mathrm{d}}-\omega L i_{\mathrm{d}} e_{\mathrm{d}}
\end{array}\right.
$$

By substituting (3) and (4) into (5), the DPC model of a three-level NPC rectifier can be expressed as:

$$
\left\{\begin{array}{l}
L \frac{\mathrm{d} p}{\mathrm{~d} t}=-E_{1} u_{\mathrm{d}}+E_{1}^{2}-R p-\omega L q \\
L \frac{\mathrm{d} q}{\mathrm{~d} t}=E_{1} u_{\mathrm{q}}-R q+\omega L p
\end{array}\right.
$$

\section{B. Neutral-Point Potential of Three-Level PWM Rectifiers}

In the high frequency mathematical model of three-level rectifiers [18], the relationship between the DC-bus neutral-point voltage variation and the switching state is obtained as:

$$
C \frac{\mathrm{d}\left(\Delta U_{\mathrm{dc}}\right)}{\mathrm{d} t}=\left[\begin{array}{lll}
S_{\mathrm{a}}^{2} & S_{\mathrm{b}}^{2} & S_{\mathrm{c}}^{2}
\end{array}\right]\left[\begin{array}{l}
i_{\mathrm{a}} \\
i_{\mathrm{b}} \\
i_{\mathrm{c}}
\end{array}\right]
$$

where $C$ is the sum of $C_{1}$ and $C_{2}$, and $\Delta U_{\mathrm{dc}}$ is the difference between the two capacitor-voltage values.

\section{INFLUENCE OF SPACE VOLTAGE VeCtORS ON ACTIVE AND REACTIVE POWER AND NEUTRAL-POINT POTENTIAL}

\section{A. Influence of Space Voltage Vectors on Active and Reactive Power}

Each voltage vector of a NPC three-level rectifier can be decomposed into $\mathrm{d}$ and $\mathrm{q}$ components. Take the first switching state as an example. Its corresponding voltage vector $\boldsymbol{u}_{1}$ projected on the d-q frame is presented in Fig. 3(a). According to Table I, the magnitude of $\boldsymbol{u}_{1}$ is $\sqrt{2 / 3} U_{\mathrm{dc}}$. Therefore, the d-q components of $\boldsymbol{u}_{1}$ can be expressed as:

$$
\begin{aligned}
& u_{1 \mathrm{~d}}=\sqrt{2 / 3} U_{\mathrm{dc}} \cos \theta \\
& u_{1 \mathrm{q}}=-\sqrt{2 / 3} U_{\mathrm{dc}} \sin \theta
\end{aligned}
$$

where $u_{1 \mathrm{~d}}$ and $u_{1 \mathrm{q}}$ are projections on the d-axis and q-axis of $\boldsymbol{u}_{1}$, respectively, and $\theta$ is the angular position of the grid voltage vector.

According to (6), it can be noted that the change rates of the active and reactive power are related to the d-axis and q-axis components of the voltage vector, respectively. The influence function of the active power $\xi$, the influence function of the reactive power $\mu$, and the parameters $m_{1}$ and $m_{2}$ are defined as:

$$
\begin{gathered}
\xi=\frac{u_{\mathrm{d}}}{\sqrt{2 / 3} U_{\mathrm{dc}}} \\
\mu=\frac{-u_{\mathrm{q}}}{\sqrt{2 / 3} U_{\mathrm{dc}}} \\
m_{1}=\frac{E_{1}^{2}-R p-\omega L q}{\sqrt{2 / 3} U_{\mathrm{dc}} E_{1}} \\
m_{2}=\frac{-R q+\omega L p}{\sqrt{2 / 3} U_{\mathrm{dc}} E_{1}}
\end{gathered}
$$

It can be noted that both $\xi$ and $\mu$ are related to the voltage vector. By substituting (8) into (9) and (10), the influence functions of the active and reactive power of the first switching state are obtained as:

$$
\begin{aligned}
& \xi_{1}=\cos \theta \\
& \mu_{1}=\sin \theta
\end{aligned}
$$

The $\xi$ and $\mu$ functions of all of the voltage vectors of NPC rectifiers are calculated based on the aforementioned contents. The results of the $\xi$ functions are shown in Fig. 4. Since the 

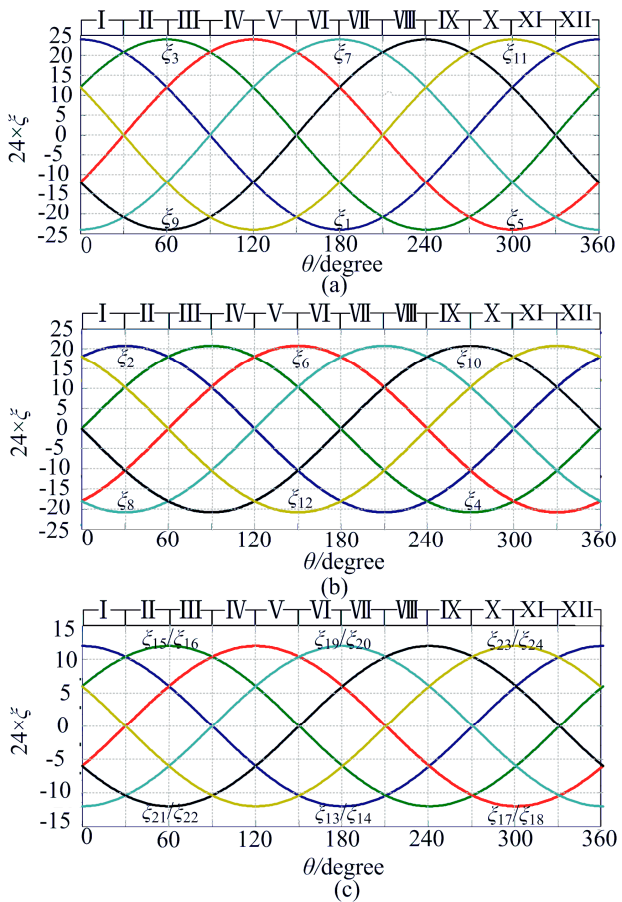

Fig. 4. Plot graphs of function $\xi$ when the space voltage vector belongs to (a) Big voltage vectors. (b) Medium voltage vectors. (c) Small voltage vectors.

TABLE II

The Relationship BETWEen ReCtifier Voltage Vector AND NeUTRAL POINT CURRENT

\begin{tabular}{|c|c|c|c|c|c|}
\hline $\boldsymbol{u}_{\mathbf{L}}$ & $i_{0}$ & $\boldsymbol{u}_{\mathbf{S}}$ & $i_{0}$ & $\boldsymbol{u}_{\mathbf{S}}$ & $i_{0}$ \\
\hline $\boldsymbol{u}_{\mathbf{2}}$ & $i_{\mathrm{b}}$ & $\boldsymbol{u}_{\mathbf{1 3}}$ & $i_{\mathrm{a}}$ & $\boldsymbol{u}_{\mathbf{1 4}}$ & $-i_{\mathrm{a}}$ \\
\hline $\boldsymbol{u}_{\mathbf{4}}$ & $i_{\mathrm{a}}$ & $\boldsymbol{u}_{\mathbf{1 5}}$ & $i_{\mathrm{c}}$ & $\boldsymbol{u}_{\mathbf{1 6}}$ & $-i_{\mathrm{c}}$ \\
\hline $\boldsymbol{u}_{\mathbf{6}}$ & $i_{\mathrm{c}}$ & $\boldsymbol{u}_{\mathbf{1 7}}$ & $i_{\mathrm{b}}$ & $\boldsymbol{u}_{\mathbf{1 8}}$ & $-i_{\mathrm{b}}$ \\
\hline $\boldsymbol{u}_{\mathbf{8}}$ & $i_{\mathrm{b}}$ & $\boldsymbol{u}_{\mathbf{1 9}}$ & $i_{\mathrm{a}}$ & $\boldsymbol{u}_{\mathbf{2 0}}$ & $-i_{\mathrm{a}}$ \\
\hline $\boldsymbol{u}_{\mathbf{1 0}}$ & $i_{\mathrm{a}}$ & $\boldsymbol{u}_{\mathbf{2 1}}$ & $i_{\mathrm{c}}$ & $\boldsymbol{u}_{\mathbf{2 2}}$ & $-i_{\mathrm{c}}$ \\
\hline $\boldsymbol{u}_{\mathbf{1 2}}$ & $i_{\mathrm{c}}$ & $\boldsymbol{u}_{\mathbf{2 3}}$ & $i_{\mathrm{b}}$ & $\boldsymbol{u}_{\mathbf{2 4}}$ & $-i_{\mathrm{b}}$ \\
\hline
\end{tabular}

values of the $\xi$ functions are very small, they are amplified 24 times in Fig. 4 to make them easier to analyze. The $\xi$ functions of the zero voltage vectors are not shown in Fig. 4 because their values are always 0 . It can be seen from Fig. 4 that the $\xi$ functions of the non-zero voltage vectors can be classified into three categories based on the magnitude of the space voltage vector and there are several characteristics of them:

1) All the $\xi$ functions are cosine functions. Therefore they are periodic functions.

2) In each category, the $\xi$ functions of the different space voltage vectors have the same magnitude, but different phase angles. For example, in the big voltage vector category, $\xi_{2}$ lags $\xi_{1}$ by $\pi / 3$, and $\xi_{3}$ lags $\xi_{2}$ by $\pi / 3$, and so on.

The results of the $\mu$ functions are very similar to those of the $\xi$ functions except that all of the $\mu$ functions are sine functions instead of cosine functions.

By substituting (9)-(12) into (6), the relationships between the influence function $\xi, \mu$ and the change rates of the active and reactive power can be expressed as:

$$
\left\{\begin{array}{l}
L \frac{\mathrm{d} p}{\mathrm{~d} t}=\sqrt{2 / 3} U_{\mathrm{dc}} E_{1}\left(m_{1}-\xi\right) \\
L \frac{\mathrm{d} q}{\mathrm{~d} t}=\sqrt{2 / 3} U_{\mathrm{dc}} E_{1}\left(m_{2}-\mu\right)
\end{array}\right.
$$

It can be noted from (15) that the relationships are proportional and can be simplified as follows:

$$
\left\{\begin{array}{l}
\frac{\mathrm{d} p}{\mathrm{~d} t} \propto\left(m_{1}-\xi\right) \\
\frac{\mathrm{d} q}{\mathrm{~d} t} \propto\left(m_{2}-\mu\right)
\end{array}\right.
$$

\section{B. Influence of Space Voltage Vectors on Neutral-Point Potential}

According to (7), the neutral point current $i_{0}$ can be expressed by:

$$
i_{0}=-\left[\begin{array}{lll}
S_{\mathrm{a}}^{2} & S_{\mathrm{b}}^{2} & S_{\mathrm{c}}^{2}
\end{array}\right]\left[\begin{array}{l}
i_{\mathrm{a}} \\
i_{\mathrm{b}} \\
i_{\mathrm{c}}
\end{array}\right]
$$

Since different values of $S_{j}\left(S_{j}=1,0\right.$ and $\left.-1, j=\mathrm{a}, \mathrm{b}, \mathrm{c}\right)$ correspond to different space voltage vectors, by substituting different values of $S_{j}$ into (17), the relationship between the space voltage vector and the neutral-point current can be obtained, as shown in Table II. Notice that only the medium voltage vectors and the small voltage vectors are related to the unbalance of the neutral-point potential. As a result, only the neutral point currents of the medium and small voltage vectors are shown in Table II.

It can be noted from (7) that the change rate of $\Delta U_{\mathrm{dc}}$ is related to the switching state and the AC currents. The neutral-point potential influence function $\delta$ is defined as:

$$
\delta=\frac{i_{0}}{|\boldsymbol{i}|}
$$

where $|\boldsymbol{i}|$ is the amplitude of the AC current vector.

As shown in Fig. 3(b), the AC current vector $\boldsymbol{i}$ can be expressed in the abc frame as:

$$
\left\{\begin{array}{l}
i_{\mathrm{a}}=|\boldsymbol{i}| \cos \alpha \\
i_{\mathrm{b}}=|\boldsymbol{i}| \cos (\alpha+2 / 3 \pi) \\
i_{\mathrm{c}}=|\boldsymbol{i}| \cos (\alpha+4 / 3 \pi)
\end{array}\right.
$$

where $\alpha$ is the angular position of the AC current vector.

According to (17)-(19), the $\delta$ functions can be expressed as:

$$
\delta=\left\{\begin{array}{cl}
\cos \alpha, & \left(i_{0}=i_{\mathrm{a}}\right) \\
\cos (\alpha+2 / 3 \pi), & \left(i_{0}=i_{\mathrm{b}}\right) \\
\cos (\alpha+4 / 3 \pi), & \left(i_{0}=i_{\mathrm{c}}\right) \\
\cos (\alpha+\pi), & \left(i_{0}=-i_{\mathrm{a}}\right) \\
\cos (\alpha+5 / 3 \pi), & \left(i_{0}=-i_{\mathrm{b}}\right) \\
\cos (\alpha+1 / 3 \pi), & \left(i_{0}=-i_{c}\right)
\end{array}\right.
$$


It can be noticed that all of the $\delta$ functions are cosine functions, and that they have characteristics similar to those of the $\xi$ and $\mu$ functions.

According to (7), (17) and (18), the relationship between the influence function $\delta$ and the change rate of $\Delta U_{\mathrm{dc}}$ can be expressed as:

$$
C \frac{\mathrm{d}\left(\Delta U_{\mathrm{dc}}\right)}{\mathrm{d} t}=-\delta|\boldsymbol{i}|
$$

It can be noted from (21) that the relationship is proportional and can be simplified as follows:

$$
\frac{\mathrm{d}\left(\Delta U_{\mathrm{dc}}\right)}{\mathrm{d} t} \propto \delta
$$

\section{PRoposed DiRECT POWER CONTROL}

\section{A. Vector Influence Tables of the Active and Reactive Power}

In order to show the values of $\xi$ and $\mu$ explicitly, two off-line vector influence tables are needed. In these tables, each value of $\xi$ or $\mu$ is not an exact value, but the average value of the small zone it belongs to. A process of discretization of the angular location of the grid voltage vector $\theta$ is carried out to divide the whole plane evenly into

12 zones. Since $\theta \in[0,2 \pi]$, each zone has $\pi / 6$ of the angular scope of $\theta$.

The average values of $\xi$ and $\mu$ in each zone are calculated as:

$$
\begin{aligned}
& f_{\xi}=\operatorname{round}\left[\frac{k}{(\pi / 6)} \int_{\frac{\pi}{6}\left(n_{\theta}-1\right)}^{\frac{\pi}{6} n_{\theta}} \xi \mathrm{d} \theta\right] \\
& f_{\mu}=\operatorname{round}\left[\frac{k}{(\pi / 6)} \int_{\frac{\pi}{6}\left(n_{\theta}-1\right)}^{\frac{\pi}{6} n_{\theta}} \mu \mathrm{d} \theta\right]
\end{aligned}
$$

where $n_{\theta}=\{1,2,3 \ldots 12\}$, round[] denotes the rounding of the number to the nearest integer, and $f_{\xi}$ and $f_{\mu}$ are the influence factors of the active power and reactive power, respectively. In order to reduce the rounding errors, a coefficient $k$ is added. When $k$ is set to 24 , the values of $f_{\xi}$ and $f_{\mu}$ are integers between -24 and +24 .

According to (16), (23) and (24), the approximate proportional relationship between $f_{\xi}, f_{\mu}$ and the change rates of the active and reactive power can be expressed as:

$$
\begin{aligned}
& \frac{\mathrm{d} p}{\mathrm{~d} t} \approx \operatorname{avg}\left(\frac{\mathrm{d} p}{\mathrm{~d} t}\right) \propto\left(k m_{1}-f_{\xi}\right) \\
& \frac{\mathrm{d} q}{\mathrm{~d} t} \approx \operatorname{avg}\left(\frac{\mathrm{d} q}{\mathrm{~d} t}\right) \propto\left(k m_{2}-f_{\mu}\right)
\end{aligned}
$$

where $\operatorname{avg}()$ denotes the average value in each zone.

By substituting (8) into (23) and (24), the values of $f_{\xi}$ and $f_{\mu}$

\begin{tabular}{|c|c|c|c|c|c|c|c|c|c|c|c|c|c|c|c|}
\hline$u_{1}$ & $\boldsymbol{u}_{2}$ & $u_{13} / u_{14}$ & 1 & 2 & 3 & 4 & 5 & 6 & 7 & 8 & 9 & 10 & 11 & 12 & \\
\hline$u_{3}$ & $u_{4}$ & $u_{15} / u_{16}$ & 3 & 4 & 5 & 6 & 7 & 8 & 9 & 10 & 11 & 12 & 1 & 2 & \\
\hline$u_{5}$ & $u_{6}$ & $u_{17} / u_{18}$ & 5 & 6 & 7 & 8 & 9 & 10 & 11 & 12 & 1 & 2 & 3 & 4 & \\
\hline 17 & $u_{8}$ & $u_{19} / u_{20}$ & 7 & 8 & 9 & 10 & 11 & 12 & 1 & 2 & 3 & 4 & 5 & 6 & \\
\hline$u_{9}$ & $u_{10}$ & $u_{21} / u_{22}$ & 9 & 10 & 11 & 12 & 1 & 2 & 3 & 4 & 5 & 6 & 7 & 8 & \\
\hline & $u_{12}$ & $u_{23} / u_{24}$ & 11 & 12 & 1 & 2 & 3 & 4 & 5 & 6 & 7 & 8 & 9 & 10 & \\
\hline & & BIG & 23 & 17 & 6 & -6 & -17 & -23 & -23 & -17 & -6 & 6 & 17 & 23 & \\
\hline & ME & EDIUM & 20 & 20 & 15 & 5 & -5 & -15 & -20 & -20 & -15 & -5 & 5 & 15 & \\
\hline & & MALL & 11 & 8 & 3 & -3 & -8 & -11 & -11 & -8 & -3 & 3 & 8 & 11 & \\
\hline
\end{tabular}
in each of the 12 zones corresponding to $\boldsymbol{u}_{1}$ are calculated. In a similar way, the values of $f_{\xi}$ and $f_{\mu}$ in each zone corresponding to other voltage vectors can also be calculated

\begin{tabular}{|c|c|c|c|c|c|c|c|c|c|c|c|c|c|c|}
\hline$u_{1}$ & $u_{2}$ & $u_{13} / u_{14}$ & 1 & 2 & 3 & 4 & 5 & 6 & 7 & 8 & 9 & 10 & 11 & 12 \\
\hline$u_{3}$ & $u_{4}$ & $u_{15} / u_{16}$ & 3 & 4 & 5 & 6 & 7 & 8 & 9 & 10 & 11 & 12 & 1 & 2 \\
\hline$u_{5}$ & $u_{6}$ & $u_{17} / u_{18}$ & 5 & 6 & 7 & 8 & 9 & 10 & 11 & 12 & 1 & 2 & 3 & 4 \\
\hline$u_{7}$ & $u_{8}$ & $u_{19} / u_{20}$ & 7 & 8 & 9 & 10 & 11 & 12 & 1 & 2 & 3 & 4 & 5 & 6 \\
\hline$u_{9}$ & $u_{10}$ & $u_{21} / u_{22}$ & 9 & 10 & 11 & 12 & 1 & 2 & 3 & 4 & 5 & 6 & 7 & 8 \\
\hline$u_{11}$ & $u_{12}$ & $u_{23} / u_{24}$ & 11 & 12 & 1 & 2 & 3 & 4 & 5 & 6 & 7 & 8 & 9 & 10 \\
\hline & & BIG & 6 & 17 & 23 & 23 & 17 & 6 & -6 & -17 & -23 & $-23-$ & -17 & -6 \\
\hline & MI & EDIUM & -5 & 5 & 15 & 20 & 20 & 15 & 5 & -5 & -15 & $-20-$ & -20 & -15 \\
\hline & & MALL & 3 & 8 & 11 & 11 & 8 & 3 & -3 & -8 & -11 & -11 & -8 & -3 \\
\hline
\end{tabular}

TABLE III

Vector Influence TABLe of Active Power

TABLE IV

Vector Influence TABLE OF REACtive POWER

and the vector influence tables of the active and reactive power are established. As shown in Table III and Table IV, the values of $f_{\xi}$ and $f_{\mu}$ are listed at the bottom of the vector influence tables. The values of $f_{\xi}$ and $f_{\mu}$ of the zero voltage vectors are not shown in Table III or Table IV because they are always 0 . The grayscale color of each small block is colored proportional to the value in the block. At the top of the table are the numbers of $n_{\theta}$ arranged in different orders in accordance with different space voltage vectors. For example, in the big voltage vector category, $\boldsymbol{u}_{3}$ lags $\boldsymbol{u}_{1}$ by $\pi / 3$. Therefore, by shifting the numbers of $n_{\theta}$ in the first row where $\boldsymbol{u}_{\mathbf{1}}$ belongs to the left by two blocks, the numbers of the second row where $\boldsymbol{u}_{3}$ belongs are obtained.

To obtain the values of $f_{\xi}$ and $f_{\mu}$ in the tables, the following steps should be followed. For example, if the value of $f_{\xi}$ of vector $\boldsymbol{u}_{\mathbf{1 6}}$ when $n_{\theta}=7$ is to be found, the specific steps are presented as follows:

Step 1: Find the voltage vector $\boldsymbol{u}_{\mathbf{1 6}}$ in the left-top area of Table III.

Step 2: In the row that vector $\boldsymbol{u}_{\mathbf{1 6}}$ belongs to, find the number 7 of $n_{\theta}$ and the column it belongs to.

Step 3: In that column, find the value in the row of the type of voltage vector at the bottom of the table. In the case of vector $\boldsymbol{u}_{\mathbf{1 6}}$, since it is a small voltage vector, the number -8 in the row of the small voltage vector is the value of $f_{\xi}$.

\section{B. Vector Influence Table of the Neutral-Point Potential}

The influence factor of the neutral-point potential $f_{\delta}$ is 
TABLE V

Vector Influence Table of Neutral Point Potential
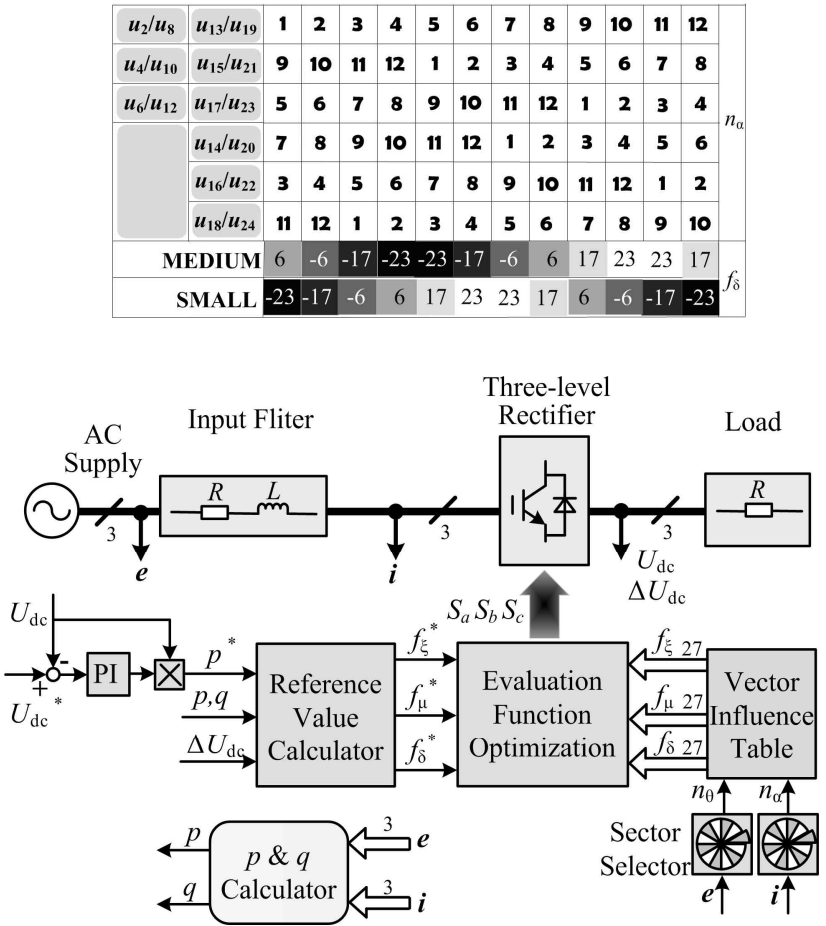

Fig. 5. Proposed DPC Block diagram.

calculated as:

$$
f_{\delta}=\operatorname{round}\left[\frac{k}{(\pi / 6)} \int_{\frac{\pi}{6}\left(n_{\alpha}-1\right)}^{\frac{\pi}{6} n_{\alpha}} \delta \mathrm{d} \alpha\right]
$$

where $n_{\alpha}=\{1,2,3 \ldots 12\}$.

According to (22) and (27), the approximate proportional relationship between $f_{\delta}$ and the change rate of $\Delta U_{\mathrm{dc}}$ can be expressed as:

$$
\frac{\mathrm{d}\left(\Delta U_{\mathrm{dc}}\right)}{\mathrm{d} t} \approx \operatorname{avg}\left(\frac{\mathrm{d}\left(\Delta U_{\mathrm{dc}}\right)}{\mathrm{d} t}\right) \propto f_{\delta}
$$

The vector influence table of the neutral-point potential can be established in a similar way, as shown in Table V. Notice that only the medium and small voltage vectors are related to the unbalance of the neutral-point potential, and only the values of $f_{\delta}$ of the medium and small voltage vectors are listed in Table V. In other words, the values of $f_{\delta}$ of the large and zero voltage vectors are always 0 . The steps to obtain the values of $f_{\delta}$ in the table are similar to the steps illustrated in Section A.

\section{Proposed DPC Block Diagram and Objective Function}

A block diagram of the proposed DPC is presented in Fig. 5. It can be seen that three vector influence tables and an objective function optimization part are used instead of the hysteresis comparators and switching table of the conventional method. The reference value of the active power $p^{*}$ is generated by a PI controller in the outer voltage loop while the reference value of the reactive power $q^{*}$ is set to 0 .
TABLE VI

SIMULATION AND EXPERIMENTAL PARAMETERS

\begin{tabular}{|c|c|}
\hline Item & Value \\
\hline AC phase voltage $/ \mathrm{V}$ & 40 \\
\hline DC bus voltage $/ \mathrm{V}$ & 120 \\
\hline AC-side inductance $/ \mathrm{mH}$ & 10 \\
\hline AC-side resistance $/ \Omega$ & 0.1 \\
\hline DC bus capacitor $/ \mu \mathrm{F}$ & 5600 \\
\hline Load resistance $/ \Omega$ & 40 \\
\hline
\end{tabular}

The values of the instantaneous active power $p$ and the reactive power $q$ can be calculated as follows [28]:

$$
\left\{\begin{array}{l}
p=e_{\mathrm{a}} i_{\mathrm{a}}+e_{\mathrm{b}} i_{\mathrm{b}}+e_{\mathrm{c}} i_{\mathrm{c}} \\
q=\frac{1}{\sqrt{3}}\left[\left(e_{\mathrm{b}}-e_{\mathrm{c}}\right) i_{\mathrm{a}}+\left(e_{\mathrm{c}}-e_{\mathrm{a}}\right) i_{\mathrm{b}}+\left(e_{\mathrm{a}}-e_{\mathrm{b}}\right) i_{\mathrm{c}}\right]
\end{array}\right.
$$

According to (25), (26) and (28), the reference values of $f_{\xi}$, $f_{\mu}$ and $f_{\delta}$ are defined as:

$$
\left\{\begin{array}{l}
f_{\xi}^{*}=-\left(p^{*}-p\right) / K_{\mathrm{i}}+k m_{1} \\
f_{\mu}^{*}=-\left(q^{*}-q\right) / K_{\mathrm{i}}+k m_{2} \\
f_{\delta}^{*}=-\Delta U_{\mathrm{dc}} / K_{\delta}
\end{array}\right.
$$

where $K_{\mathrm{i}}$ and $K_{\delta}$ are parameters which can be calculated by:

$$
\left\{\begin{array}{l}
K_{\mathrm{i}}=\frac{\sqrt{2 / 3} U_{\mathrm{dc}} T_{\mathrm{s}} E_{1}}{k L} \\
K_{\delta}=\frac{T_{\mathrm{s}}|i|}{k C}
\end{array}\right.
$$

where $T_{\mathrm{s}}$ is the sampling period.

In order to achieve power tracking and neutral point balance at the same time, the optimized space voltage vector should be selected so that the values of $f_{\xi}, f_{\mu}$ and $f_{\delta}$ can approach as near as possible to their reference values of $f_{\xi}^{*}$, $f_{\mu}^{*}$ and $f_{\delta}^{*}$. Therefore, the objective function can be described as:

$$
g=\left|f_{\xi}^{*}-f_{\xi}\right|+\left|f_{\mu}^{*}-f_{\mu}\right|+\lambda_{\delta}\left|f_{\delta}^{*}-f_{\delta}\right|
$$

where $\lambda_{\delta}$ is the weighting factor. The objective function $g$ is calculated for each set of values of $f_{\xi}, f_{\mu}$ and $f_{\delta}$ corresponding to the 27 space voltage vectors. The space voltage vector that corresponds to the minimal value of $g$ will be selected and applied during the next control period. By giving $\lambda_{\delta}$ different values, the priorities of the power control and the neutral point balance control can be made differently.

\section{Simulation RESUlts}

The performances of the conventional two-level hysteresis DPC method and the proposed DPC method are given and compared by Matlab/Simulink. The simulation parameters are presented in Table VI.

The dynamic performances of the conventional and 


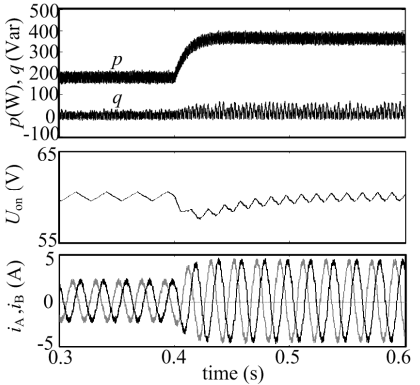

(a)

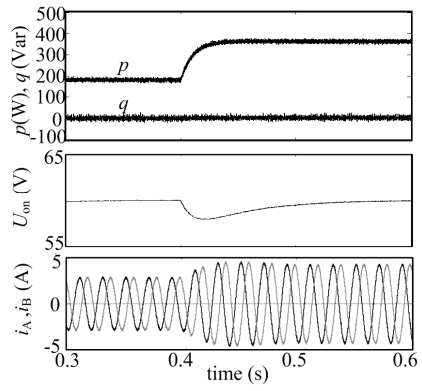

(b)
Fig. 6. Simulation result of dynamic performance. (a) active and reactive power, neutral-point potential and $\mathrm{AC}$ currents for the conventional method. (b) active and reactive power, neutral-point potential and AC currents for the proposed method.

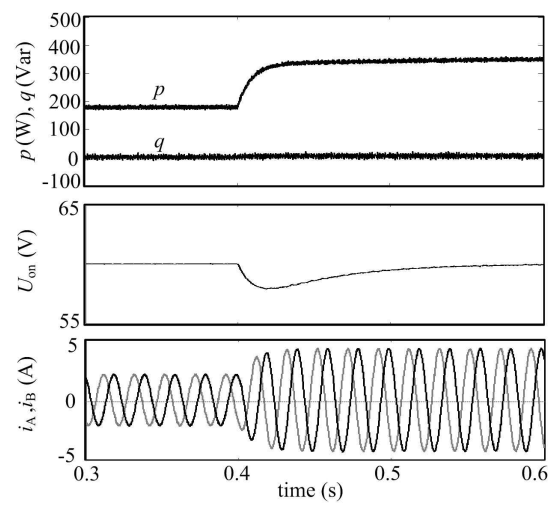

Fig.7. Simulation result of active and reactive power, neutral-point potential and AC currents for the proposed method with $1.5 L$

proposed methods are shown in Fig. 6, where an abrupt change of load (from $80 \Omega$ to $40 \Omega$ ) is introduced at $t=0.4 \mathrm{~s}$.

It can be seen that both the conventional and the proposed methods have a fast dynamic response. However, the conventional method causes larger power and neutral point potential ripples and a seriously distorted AC current, which are greatly improved by the proposed method. To further illustrate and validate the improvements of the proposed method, the standard deviations of the active and reactive power $\sigma_{\mathrm{p}}$ and $\sigma_{\mathrm{q}}$ and the neutral point potential $\sigma_{\text {npp }}$ are calculated. The standard deviation of a generic variable $x$ is expressed as:

$$
\sigma_{x}=\sqrt{\frac{1}{n-1} \sum_{i=1}^{n}\left(x_{\mathrm{i}}-\bar{x}\right)^{2}}
$$

where $n$ is the number of samples, and $\bar{x}=\frac{1}{n} \sum_{i=1}^{n} x_{\mathrm{i}}$

According to the calculated results, $\sigma_{\mathrm{p}}$ and $\sigma_{\mathrm{q}}$ of the conventional method during the time period of $0.55 \mathrm{~s}$ to $0.6 \mathrm{~s}$ are $15.22 \mathrm{~W}$ and $19.85 \mathrm{Var}$, while $\sigma_{\mathrm{p}}$ and $\sigma_{\mathrm{q}}$ of the proposed method are only $4.86 \mathrm{~W}$ and 6.06 Var. The standard deviations of the active and reactive power are reduced by $68.07 \%$ and $69.47 \%$, respectively. In addition, the values of $\sigma_{\text {npp }}$ of the

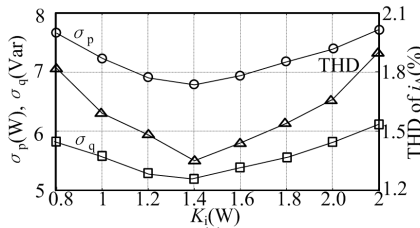

(a)

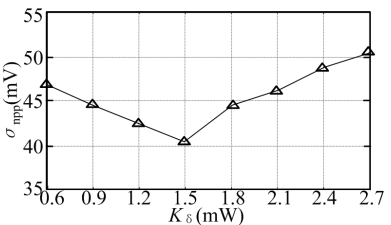

(b)
Fig. 8. Simulation results with the variation of $K_{\mathrm{i}} 、 K_{\delta}$. (a) $\sigma_{\mathrm{p}}, \sigma_{\mathrm{q}}$ and THD of $i_{\mathrm{A}}$ with the variation of $K_{\mathrm{i}}$ (b) $\sigma_{\mathrm{npp}}$ with the variation of $K_{\delta}$.

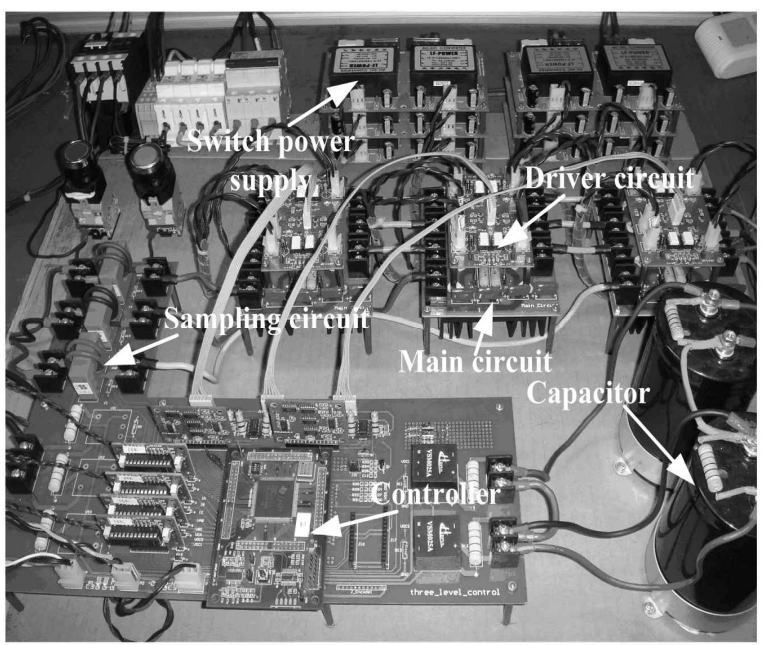

Fig. 9. Photograph of experimental setup.

conventional method and the proposed method are $292.9 \mathrm{mV}$ and $40.5 \mathrm{mV}$, respectively. The ripple size of the neutral point potential is reduced by $86.17 \%$.

In Fig. 7, the robustness against system parameter variations is tested for the proposed method. In the simulation, the $d-q$ inductances are increased to $15 \mathrm{mH}(150 \%$ of their nominal values), and the other simulation conditions are the same as those in Fig. 6. It can be seen that the variation of line inductance does not have a significant effect on system performance with the values of $\sigma_{\mathrm{p}}$ and $\sigma_{\mathrm{q}}$ being $5.02 \mathrm{~W}$ and 6.16Var.

Fig. 8 shows the standard deviations of the active and reactive power, the total harmonic distortion (THD) of the $\mathrm{AC}$ currents and the standard deviation of the neutral point potential of the proposed method with different $K_{\mathrm{i}}$ and $K_{\delta}$. According to (31) and the parameters listed in Table VI, $E_{1}$ is estimated to be $69 \mathrm{~V},|\boldsymbol{i}|$ is estimated to be $4 \mathrm{~A}$, and the theoretical values of $K_{\mathrm{i}}$ and $K_{\delta}$ can be calculated as $1.4 \mathrm{~W}$ and $1.5 \mathrm{mV}$, respectively. According to (11) and (12), $m_{1}$ and $m_{2}$ are estimated to be 0.70 and 0.16 , respectively. It can be seen from Fig. 8(a) that when $K_{\mathrm{i}}$ changes within a range between $0.8 \mathrm{~W}$ and $2 \mathrm{~W}$, the values of $\sigma_{\mathrm{p}}$ and $\sigma_{\mathrm{q}}$ remain within a range between $4 \mathrm{~W} / \mathrm{Var}$ and $8 \mathrm{~W} / \mathrm{Var}$, and the THDs of the AC currents remain within a range between $1.2 \%$ and $2.1 \%$. It can also be seen that both $\sigma_{\mathrm{p}}$ and $\sigma_{\mathrm{q}}$ and the THD of the AC currents are minimal when $K_{\mathrm{i}}$ is approximately $1.4 \mathrm{~W}$, which is consistent with the theoretical value of $K_{\mathrm{i}}$. It can be seen 


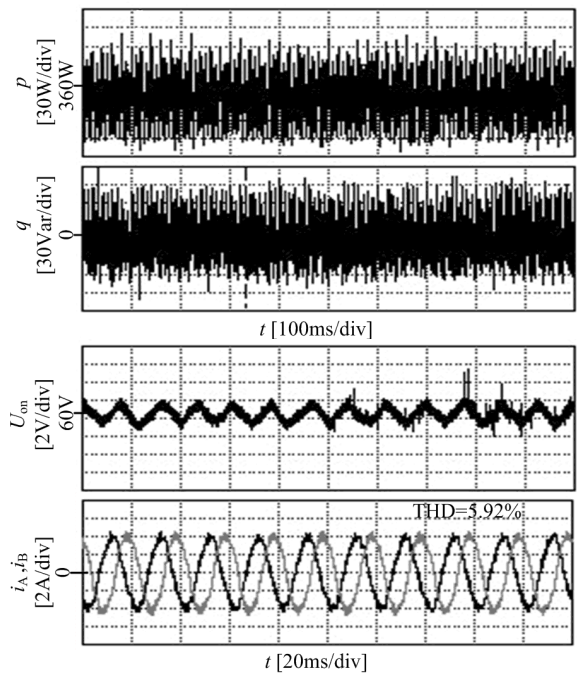

(a)

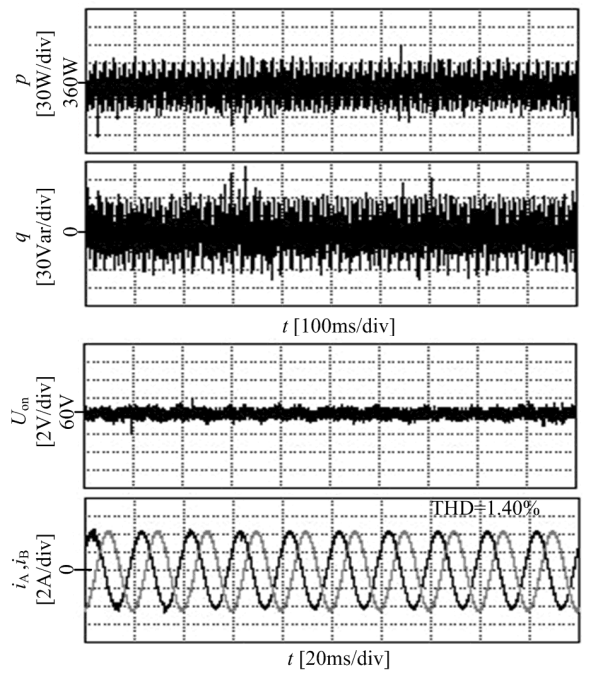

(b)

Fig. 10. Experimental waveforms for steady-state performance of active and reactive power, neutral point potential and AC currents with the load being $40 \Omega$. (a) the conventional method. (b) the proposed method.

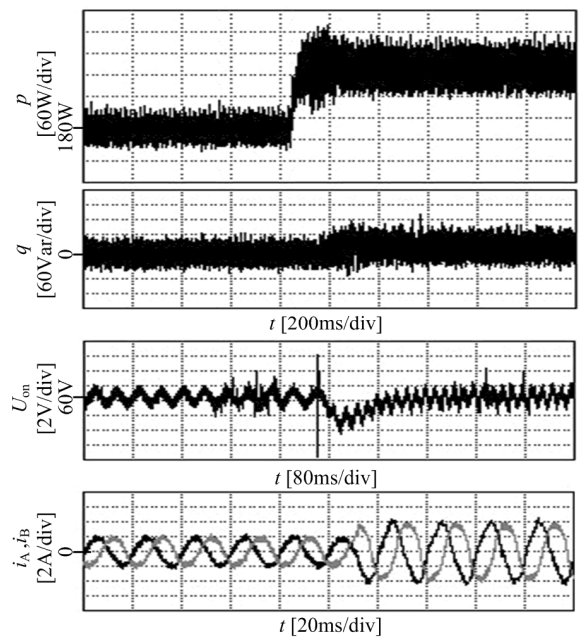

(a)

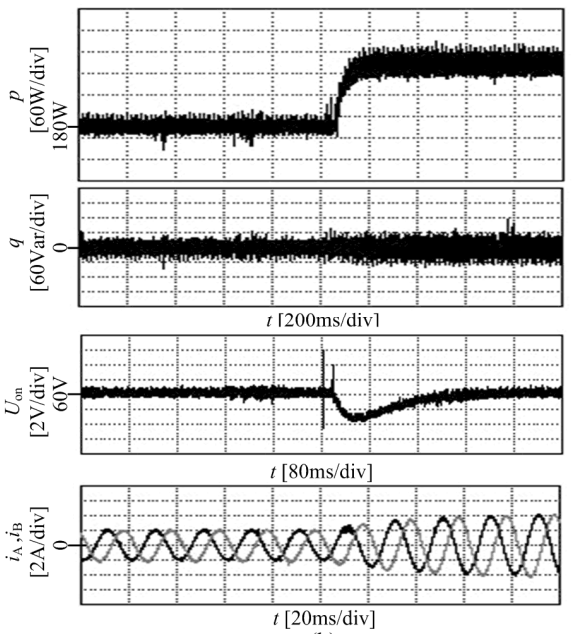

(b)

Fig. 11. Experimental waveforms of active and reactive power, neutral point potential and AC currents for step change of load from $80 \Omega$ to $40 \Omega$. (a) the conventional method. (b) the proposed method.

from Fig. 8(b) that when $K_{\delta}$ changes within a range between $0.6 \mathrm{mV}$ and $2.7 \mathrm{mV}$, the values of $\sigma_{\text {npp }}$ are within a range between $35 \mathrm{mV}$ and $55 \mathrm{mV}$, and $\sigma_{\text {npp }}$ is minimal when $K_{\delta}$ is approximately $1.5 \mathrm{mV}$, which is consistent with the theoretical value of $K_{\delta}$.

\section{EXPERIMENTAL RESULTS}

To verify the feasibility and effectiveness of the proposed method, a three-level rectifier prototype was implemented, as shown in Fig. 9. The system consists of a main circuit, a control circuit, a driver circuit and a sampling circuit. The complete system was connected to the utility grid through a three-phase variac. The control system employed a TMS320F28335 digital signal processor for the control strategy. The sampling periods of the conventional and proposed methods are both $50 \mu \mathrm{s}$. The parameters of the experimental system are presented in Table VI. According to section $\mathrm{V}, K_{\mathrm{i}}$ and $K_{\delta}$ are chosen as $1.4 \mathrm{~W}$ and $1.5 \mathrm{mV}$, respectively, and $m_{1}$ and $m_{2}$ are chosen to be 0.70 and 0.16 , respectively.

\section{A. Steady-State Performance}

The steady-state performances of the two methods are presented in Fig. 10 with the load being $40 \Omega$ and the AC-side inductance being $10 \mathrm{mH}$. It is clearly seen that the conventional DPC method results in significant current harmonic distortions as well as power and neutral point potential ripples, while the proposed DPC method has achieved a greatly improved performance with smaller ripples of the active and reactive powers, a well-smoothed neutral point potential and more sinusoidal AC currents. To 


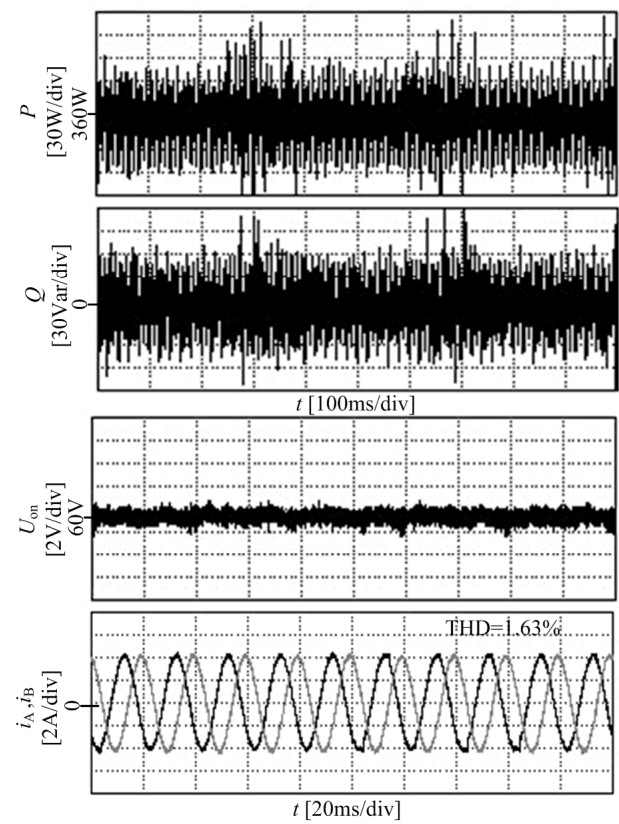

(a)

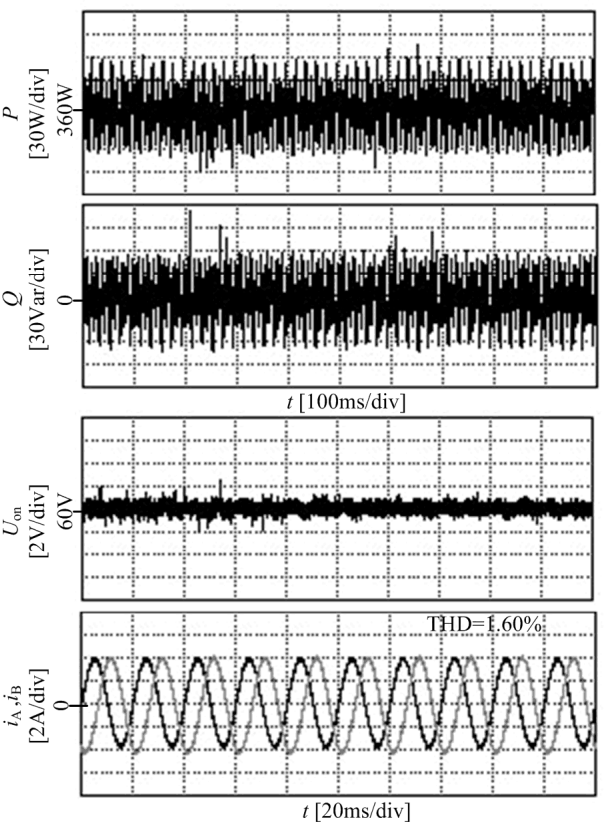

(b)

Fig. 12. Experimental waveforms of active and reactive power, neutral point potential and AC currents for the proposed method with variations of the line inductance. (a) $L=8 \mathrm{mH}$. (b) $L=12 \mathrm{mH}$

be specific, with the conventional method, as shown in Fig. $10(\mathrm{a}), \sigma_{\mathrm{p}}$ and $\sigma_{\mathrm{q}}$ are $30.37 \mathrm{~W}$ and $26.78 \mathrm{Var}$, while with the proposed method, as shown in Fig. 10(b), $\sigma_{\mathrm{p}}$ and $\sigma_{\mathrm{q}}$ are only $15.17 \mathrm{~W}$ and $20.35 \mathrm{Var}$. The percentages of the standard deviation reduction of the active and reactive power are $50.05 \%$ and $24.01 \%$, respectively. The THD of the AC currents is further calculated. The result shows that the THDs of the AC currents of the conventional and proposed methods are $5.92 \%$ and $1.40 \%$, respectively. In addition, the ripple size of the neutral point potential is reduced by $69.60 \%$ since the values of $\sigma_{\text {npp }}$ of the conventional method and proposed method are $0.556 \mathrm{~V}$ and $0.169 \mathrm{~V}$, respectively.

\section{B. Dynamic Performance}

The dynamic performances of the two methods are presented in Fig. 11 with load stepping from $80 \Omega$ to $40 \Omega$. Once again, the improvements are evident in the case of the proposed DPC method. It can be seen that with an abrupt change of load, for both the conventional and the proposed methods, the active and reactive power and the neutral point potential respond rapidly and meet the reference values only after a short period. However, remarkable ripple reductions of the active and reactive power and the neutral point potential can be observed in the proposed method.

\section{Impact of the Variations of the Line Inductance}

Further tests on the impact of line inductance variations on the steady-state performance with the proposed DPC strategy are carried out. Fig. 12 shows the waveforms of the active and reactive power, the neutral point potential and the $\mathrm{AC}$ currents of the proposed method when there are errors in the
AC-side inductance parameter. As shown in Fig. 12(a), with the line inductance $L$ being $8 \mathrm{mH}$ while the other experimental conditions are the same as those in Fig. $10(\mathrm{~b}), \sigma_{\mathrm{p}}$ and $\sigma_{\mathrm{q}}$ of the proposed method become $25.81 \mathrm{~W}$ and $23.35 \mathrm{Var}$, and the THD of the AC currents becomes 1.63\%. As shown in Fig. 12(b), with the line inductance $L$ being $12 \mathrm{mH}$ while the other experimental conditions are the same as those in Fig. 10(b), $\sigma_{\mathrm{p}}$ and $\sigma_{\mathrm{q}}$ of the proposed method become $25.28 \mathrm{~W}$ and 24.56Var, and the THD of the AC currents becomes $1.60 \%$. In both cases, the ripple sizes of the neutral point potential remain small. It can be seen that such line inductance errors have no significant influence on system performance.

\section{CONCLUSIONS}

In this paper, a novel DPC algorithm has been proposed for NPC three-level rectifiers. This paper defines the influence functions to analyze the relationships between the voltage vectors of the NPC rectifiers and the change rates of the active power, the reactive power and the neutral point potential. By discretizing and averaging the influence functions, the influence factors of the active power, the reactive power and the neutral point potential $f_{\xi}, f_{\mu}$ and $f_{\delta}$ are obtained, and they have approximately proportional relationships with the change rates of the active power, the reactive power and the neutral point potential, respectively. Furthermore, three pre-calculated vector influence tables are established based on the influence factors, and by minimizing an objective function an appropriate vector can be selected. The performance of the novel DPC has been verified by simulation and experimental results. Compared to the 
conventional DPC method, remarkable ripple reductions of the active and reactive power and the neutral point potential can be achieved by the proposed method.

\section{ACKNOWLEDGMENT}

The authors are grateful to the National Key Basic Research Program of China (2013CB035602), and the Research Fund for the Doctoral Program of Higher Education of China (20110032120082) for supporting this work.

\section{REFERENCES}

[1] C. L. Xia, X. Gu, T. N. Shi, and Y. Yan, "Neutral-point potential balancing of three-level inverters in direct-driven wind energy conversion system," IEEE Trans. Energy Conver., Vol. 26, No. 1, pp. 18-29, Mar. 2011.

[2] D. M. Lee, J. W. Jung, and S. S. Kwak, "Simple space vector PWM scheme for 3-level NPC inverters including the overmodulation region," Journal of Power Electronics, Vol. 11, No. 5, pp. 688-696, Sep. 2011.

[3] Z. B. Ye, Y. M. Xu, F. Li, X. M. Deng, and Y. Z. Zhang, "Simplified PWM strategy for neutral-point-clamped (NPC) three-level converter," Journal of Power Electronics, Vol. 14, No. 3, pp. 519-530, May 2014.

[4] N. Li, Y. Wang, W. J. Lei, R. G. Niu, and Z. A. Wang, "Novel carrier-based pwm strategy of a three-level NPC voltage source converter without low-frequency voltage oscillation in the neutral point," Journal of Power Electronics, Vol. 14, No. 3, pp. 531-540, May 2014.

[5] J. Zaragoza, J. Pou, S. Ceballos, E. Robles, P. Ibaez, and J. L. Villate, "A comprehensive study of a hybrid modulation technique for the neutral-point-clamped converter," IEEE Trans. Ind. Electron., Vol. 56, No. 2, pp. 294-304, May 2009.

[6] Y. C. Zhang, Z. M. Zhao, and J. G. Zhu, "Hybrid PWM applied to high-power three-level inverter-fed induction-motor drives," IEEE Trans. Ind. Electron., Vol. 58, No. 8, pp. 3409-3420, Aug. 2011.

[7] W. S. Song, X. Y. Feng, and C. L. Xiong, "a neutral point voltage regulation method with SVPWM control for single-phase three-level NPC converters," IEEE Vehicle Power and Propulsion Conference, pp. 1-4, 2008.

[8] N. Li, Y. Wang, S. Li, Y. F. Li, and Z. A. Wang, "Direct power control strategy used in three-level NPC converters", International Power Electronics and Motion Control Conference, pp. 1675-1679, 2012.

[9] C. Ortega, A. Arias, C. Caruana, J. Balcells, and G. Asher, "Improved waveform quality in the direct torque control of matrix-converter-fed PMSM drives," IEEE Trans. Ind. Electron., Vol. 57, No. 6, pp. 2101-2110, Jun. 2010.

[10] M. P. Kazmierkowski and L. Malesani, "Current control techniques for three-phase voltage-source PWM converters: A survey," IEEE Trans. Ind. Electron., Vol. 45, No. 5, pp. 691-703, Oct. 1998.

[11] W. Chen, Y. P. Zou, L. J. Xu, "Direct power control for neutral-point-clamped three-level PWM rectifier," Proceedings of the IEEE International Conference on Industrial Technology, pp. 1-6, 2008.

[12] L. A. Serpa, P. M. Barbosa, P. K. Steimer, and J. W. Kolar, "Five-level virtual-flux direct power control for the active neutral-point clamped multilevel inverter," IEEE Annual Power Electronics Specialists Conference, pp.
1668-1674, 2008

[13] A. Ejlali and D. Arab Khaburi, "Power quality improvement using nonlinear-load compensation capability of variable speed DFIG based on DPC-SVM method," Power Electronics, Drive Systems and Technologies Conference, pp. 280-284, 2014.

[14] L. Xiao, S. D. Huang, T. Liu, Q. Xu, and K. Y. Huang, “A novel SVM-DPC control method for grid connected AC-DC converters under asymmetrical fault," International Conference on Power Engineering, Energy and Electrical Drives, pp. 1-6, 2011.

[15] L. Xiao, S. D. Huang, L. Zhen, Q. Xu, K. Y. Huang, "Sliding mode SVM-DPC for grid-side converter of D-PMSG under asymmetrical fault," International Conference on Electrical Machines and Systems, pp. 1-6, 2011.

[16] J. Rodriguez, J. Pontt, J. Correa, P. Lezana, and P. Cortes, "Predictive power control of an AC/DC/AC converter," In Proceedings of IEEE Industry Applications Conference, 40th IAS Annual Meeting, pp. 934-939, 2005.

[17] P. Cortes, J. Rodriguez, P. Antoniewicz, and M. Kazmierkowski, "Direct power control of an AFE using predictive control," IEEE Trans Power Electron., Vol. 23, No. 5, pp.2516-2523, Sep. 2008.

[18] W. J. Wu, Y. R. Zhong, and B. Duan, "A symmetrical three-section PWM for three-level rectifier with medium vectors being eliminated," Transactions of China Electrotechnical Society, Vol. 26, No. 4, pp.69-76+94, Jun. 2009.

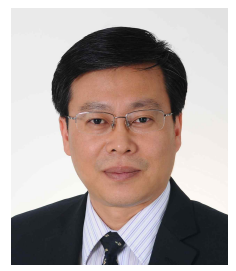

Chang-Liang Xia was born in Tianjin, China, in 1968. He received his B.S. degree from Tianjin University, Tianjin, China, in 1990, and his M.S. and Ph.D. degrees from Zhejiang University, Hangzhou, China, in 1993 and 1995, respectively, all in Electrical Engineering. He is currently a Professor in the School of Electrical Engineering and Automation, Tianjin University, and also in the Tianjin Key Laboratory of Advanced Technology of Electrical Engineering and Energy, Tianjin Polytechnic University, Tianjin, China. In 2008, he became a "Yangtze Fund Scholar" Distinguished Professor and is currently supported by the National Science Fund for Distinguished Young Scholars. His current research interests include electrical machines and their control systems, power electronics, and the control of wind generators.

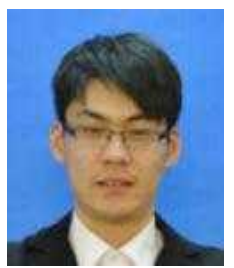

Zhe Xu was born in Handan, Hebei, China, in 1988. He received his B.S. and M.S. degrees in Electrical Engineering from Tianjin University, Tianjin, China, in 2011, 2014, respectively. His current research interests include control systems and power electronics.

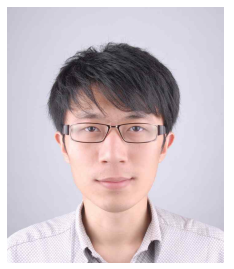

Jia-Xin Zhao was born in Tianjin, China, in 1984. He received his B.S. degree from the China Agricultural University, Beijing, China, in 2006, and his M.S. and Ph.D. degrees from Tianjin University, Tianjin, China, in 2010 and 2014, respectively, all in Electrical Engineering. He is currently an Embedded Software Engineer for REnergy Electric Tianjin Ltd., Tianjin, China. His current research interests include electrical machines and motor drives, power electronics and wind power technology. 